\title{
Role of Radiologist in Pre Surgical Evaluation of Perianal Fistula
}

\author{
Kumar Ashok Charan', Srikanth Vankineni², Parthasarthy K R ${ }^{3}$, Mehathab M Bava ${ }^{4}$, Arpit Pandey \\ ${ }^{1}$ Assistant Professor, Department of Radiodiagnosis, ${ }^{2}$ Assistant Professor, Department of Radiodiagnosis, ${ }^{3}$ Professor and \\ Head, Department of Radiodiagnosis, ${ }^{4}$ Junior Resident, Department of Radiodiagnosis, ${ }^{5}$ Junior Resident, Department of \\ Radiodiagnosis, SSIMS and RC, Davangere and Dr B R Ambedkar Medical Colleg, Bengaluru, India
}

Corresponding author: Dr Srikanth Vankineni, Assistant Professor, Department of Radiodiagnosis, SSIMS and RC, Davangere, Karnataka 577004, India

DOI: http://dx.doi.org/10.21276/ijcmsr.2019.4.3.33

How to cite this article: Kumar Ashok Charan, Srikanth Vankineni, Parthasarthy K R, Mehathab M Bava, Arpit Pandey. Role of radiologist in pre surgical evaluation of perianal fistula. International Journal of Contemporary Medicine Surgery and Radiology. 2019;4(3):C152-C157.

\section{A B S T R A C T}

Introduction: A fistula-in-ano is an abnormal hollow tract or cavity that is lined with granulation tissue and that connects a primary opening inside the anal canal to a secondary opening in the perianal skin; secondary tracts may be multiple and can extend from the same primary opening. Radiograph and Computed tomography has become obsolete investigation. In this study we gave an outline of classification of perianalfistulae and present a pictorial assay of sphincter anatomy and USG, MRI findings inperianal fistulae.

Material and methods: This prospective study had been carried out with main source of data for the study were patients from S.S. Institute of medical sciences Davangere and clinical inputs, illustrations from Dr.B.R. Ambedkar Medical college, Bangalore. This study is based on prospective analysis of 40 patients with clinical diagnosis of perianal fistulae.

Results: In the present study, On Radiological evaluation we found that - In 40cases 32 patients had single tract (80\%), 8 cases had multiple tracts (20\%). 38 cases(95\%) had single external opening, rest of 2 cases (5\%) multiple external opening. $33(82.2 \%)$ cases shows ramification. 26 cases (65\%) intersphincteric fistulae seen. 12cases (30\%) Transphincteric fistulae seen. 2 cases (5\%) extrasphincteric fistulae seen. The 8 cases were presented with swelling.

Conclusion:Radiograph and computed tomography do not provide much details and can be consider as obsolete investigation. The endo-anal ultrasound approach was entirely helpful for evaluating the sphincteric course of fistula, but gives no mind about the extrasphincteric course which made magnetic resonance imaging more acceptable technique for evaluating perianal fistula since it presents an estimate about the extrasphincteric course. MR imaging provides precise location of the fistulous track, and its relationship to pelvic floor and the sphincter complex and helps in the identification of secondary tracks and abscesses. Hence forth the present study showed that MR imaging provides precise location of the fistulous track, and its relationship to pelvic floor and the sphincter complex and helps in the identification of secondary tracks and abscesses

Keywords: Fistulogrpahy; Magnetic Resonance Imaging; Intersphincteric Fistulae; Transphincteric Fistulae; Extrasphincteric Fistulae; Suprasphincteric Fistulae; Ramification

\section{INTRODUCTION}

Fistula in ano is a common condition defined by an abnormal perianal track that connects two epithelialized surfaces, usually the anal canal to the perianal skin. Some fistulas have a tendency to recur, despite seemingly curative surgery, and recurrence rates may reach $25 \%$. Successful surgical management of anal fistulas requires an accurate preoperative assessment of the primary fistula's course and the affected pelvic structures. Transperineal ultrasound should be performed as first-line imaging modality for suspicion of low perianal fistula with high sensitivity, specificity, and NPV at much lower cost and less time as compared to perianal MRI.The advantages of magnetic resonance (MR) imaging include multiplanar imaging and a high degree of soft tissue differentiation. The primary importance of MR imaging lies in its ability to demonstrate hidden areas of sepsis and secondary extensions. In this study we gave an outline of classification of perianalfistulae and present a pictorial assay of sphincter anatomy and USG, MRI findings inperianal fistulae.

\section{MATERIAL AND METHODS}

This prospective observational study was carried out on 80 patients in SS institute of medical sciences, Davangere, Karnataka with main source of data for the study were patients from S.S. Institute of medical sciences Davangere and clinical inputs, illustrations from Dr. B.R. Ambedkar Medical college, Bangalore. The study period was 2 years (May 2017 to May 2019). This study was based on prospective analysis of 40 patients with clinical diagnosis of perianal fistulae. This MRI preoperative analysis of perianal fistulae revealed total 40 patients with fistulous tract. The principle objective of this study was to classify the perianal 
fistulae and delineate ramification which helps surgeons to operate the fistulae which will reduce the recurrence. The study population was drawn from patients who presented to SS institute of medical sciences, Davangere with Perianal discharge and Pain and underwent radiological evaluation from May 2017 to May 2019.

\section{Inclusion criteria}

1. Patients with perianal discharge and pain

2. Either sex

\section{Exclusion criteria}

1. Pregnant women.

2. Patient having history of claustrophobia.

3. Patient having history of metallic implants insertion,

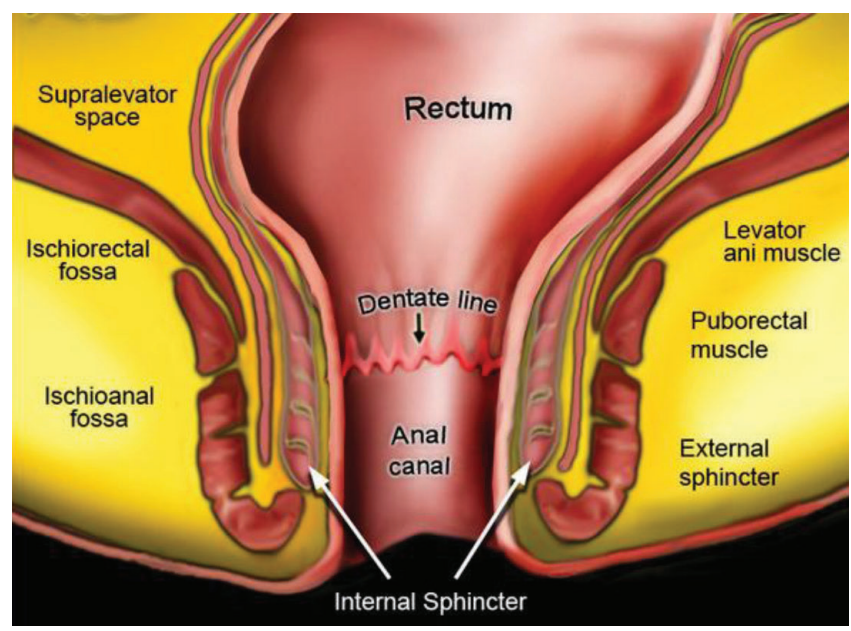

Figure-1: Graphical representation of anal canal cardiac pacemakers and metallic foreign body insitu.

4. Patients who are physically inactive.

\section{Procedure methodology}

After written informed consent was obtained were subjected to Radiograph Fistulogrpahy, endoanal ultrasound, transperineal using high frequency mechanical transrectal rotating probe for endoanal approach, high frequency superficial linear probe (5 to $12 \mathrm{Mhz}$ ) for transperineal approach and MR imaging at our institution were carried out on a 1.5-T MRI system (GE Signa Excite 1.5T) using an 8-channel phased-array coil. The sequences evaluated were:

\section{RESULT}

In the present study, on Radiological evaluation we found that - in 40 cases 32 patients had single tract (80\%), 8

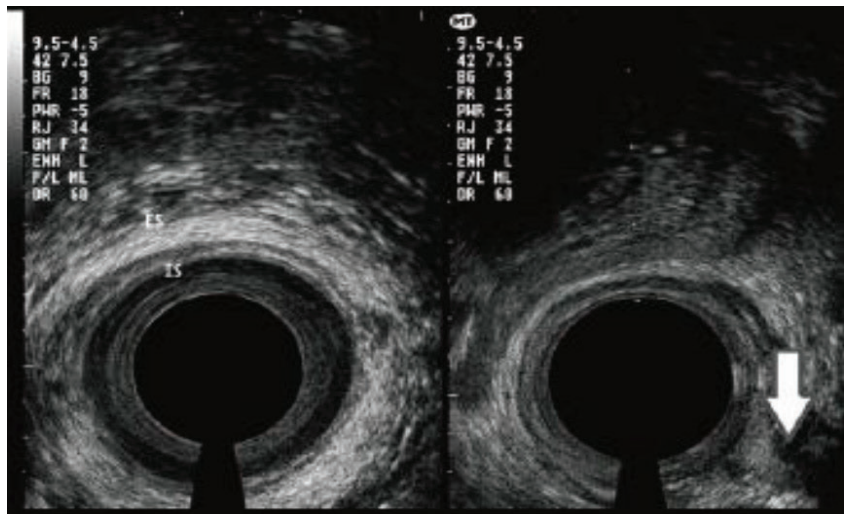

Figure-3:
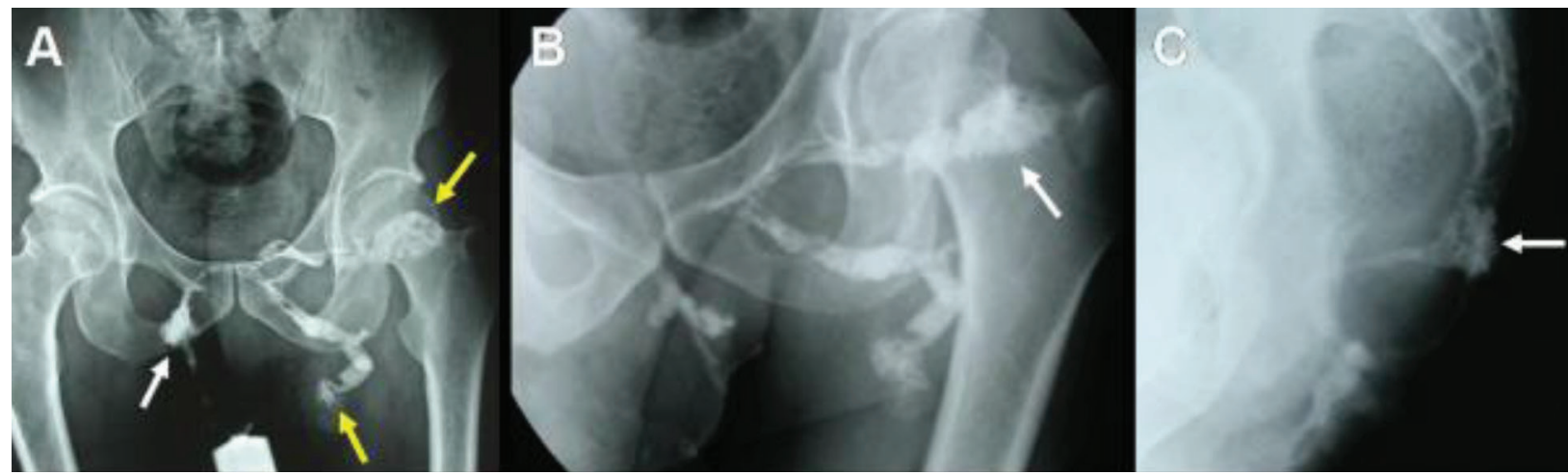

Figure-2: (A) Antero-posterior aspect, the contrast agent was given through the external opening at the right-side of the anal channel, note the course of horseshoe fistula tracts; there was a complete arm at the right side (white arrow), while the leftside arms were blind (yellow arrows), (B) Oblique aspect, the blind arm of the horseshoe fistula extended into the left gluteal area (arrow),(C)Lateral aspect, the connection between the fistula tract and the deep post anal space (arrow) was easily shown.

\begin{tabular}{l|l|c|c|c|c|}
\hline Sequence & Plane & TR/TE & FOV & Slice Thickness & Matrix \\
\hline T2W FSE & Sagittal & $3500 / 120$ & $26 \times 26$ & 3 & $512 \times 320$ \\
\hline T1W FSE & Oblique axial & $750 / 15$ & $30 \times 30$ & 4 & $320 \times 256$ \\
\hline T2W FSE & Oblique axial & $3500 / 140$ & $28 \times 28$ & 4 & $512 \times 320$ \\
\hline FS T1W FSE & Oblique axial & $700 / 20$ & $30 \times 30$ & 4 & $512 \times 256$ \\
\hline LAWA & Oblique axial & $750 / 15$ & $35 \times 35$ & 4 & $512 \times 256$ \\
\hline STIR & Coronal & $3500 / 35$ & 4 & $512 \times 512$ \\
\hline $\begin{array}{l}\text { Suggested Protocol for MR Imaging of Perianal Fistulas } \\
\text { Note FOV = field of view, FS = fat-suppressed, FSE = fast spin-echo, LAVA = liver acquisition with volume acceleration, }\end{array}$ \\
T1W = T1-weighted, T2W = T2-weighted, TE = echo time, TR = repetition time
\end{tabular}


cases had multiple tracts (20\%). 38 cases(95\%) had single external opening, rest of 2 cases (5\%) multiple external opening. 33(82.2\%) cases shows ramification. 26 cases (65\%)

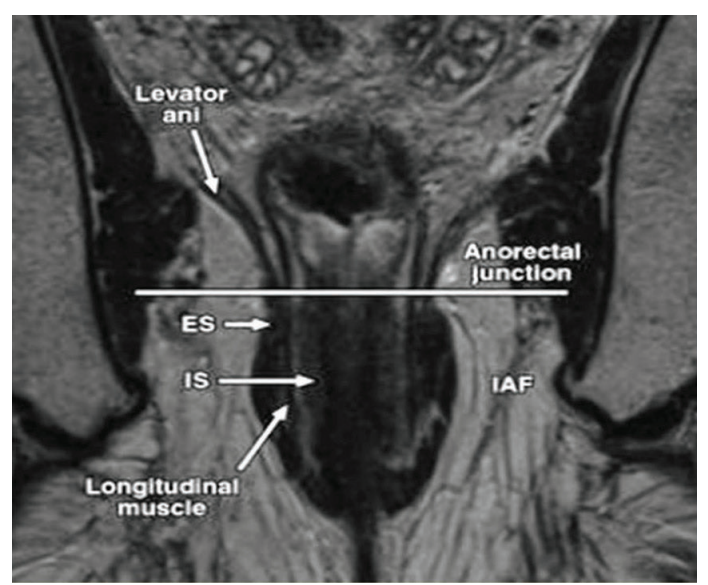

Figure-4: Coronal t 2 weighted image that corresponds to ischioanalfossa (iaf), external sphincter (es), and internal sphincter(is)

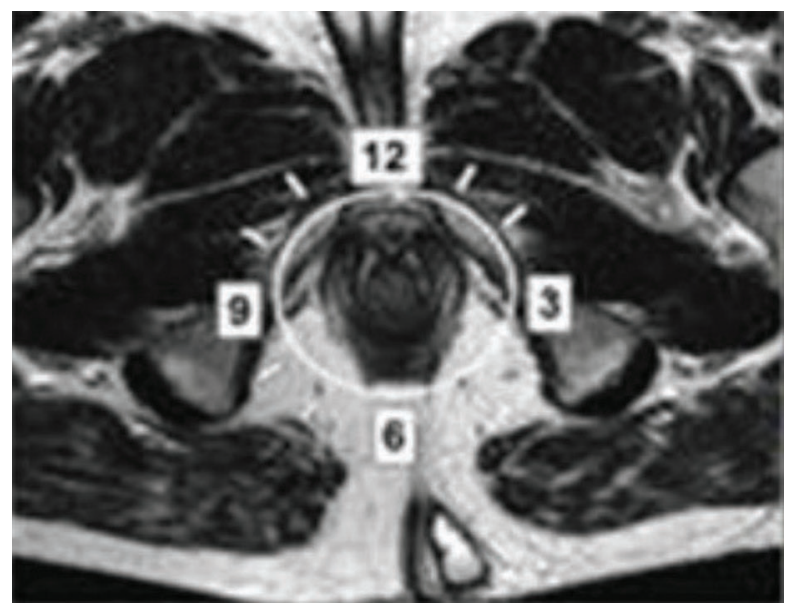

Figure-5: $12 \mathrm{~h}$ anterior perineum, $6 \mathrm{~h}$ natal cleft, $3 \mathrm{~h}$ left lateral aspect, $9 \mathrm{~h}$ right lateral aspect

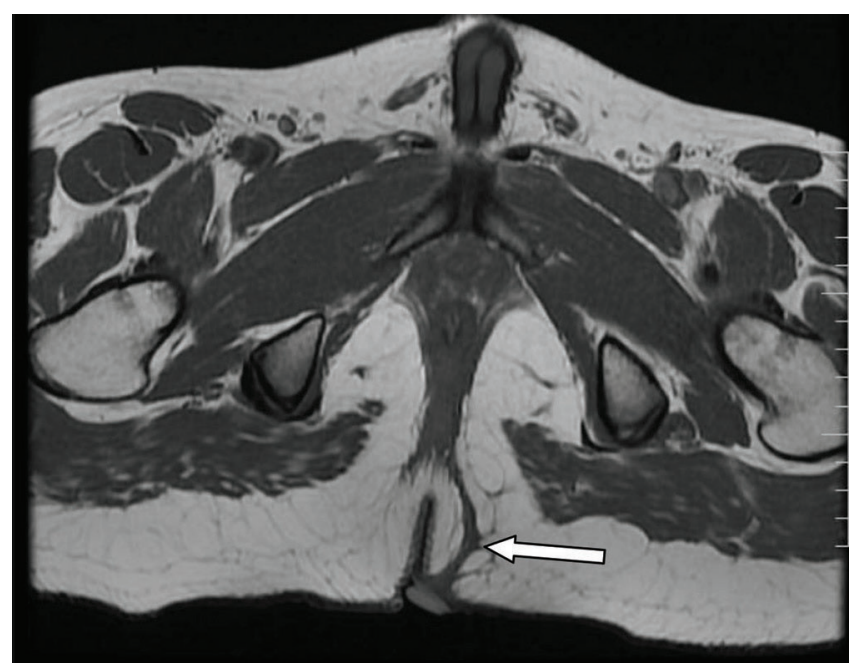

Figure-6: Intersphinteric perianal fistula measuring $6.2 \mathrm{~cm}$ in length with cutaneous opening in the left gluteal region at 6' $\mathrm{O}$ clock position and internal anal opening at 6'O clock position. intersphincteric fistulae seen. 12cases (30\%) Transphincteric fistulae seen. 2 cases (5\%) extrasphincteric fistulae seen. The 8 cases were presented with swelling. The mean age on present study was $34(\mathrm{Sd}+) 2.3$ years. The youngest case was 21 years and elder was 61 years. The most cases were in $26-45$ years.

\section{DISCUSSION}

As multiple medical and surgical treatment options exist, imaging plays a critical role in accurately characterizing perianal fistulas to individualize management strategy. Differences in the classification scheme have been shown to have an impact on prediction of prognosis. Imaging options include fistulography, computed tomography (CT), anal endosonography, and MRI.

\section{Anatomy}

Image interpretation depends on knowledge of anatomy of anal canal and anal sphincters. The anal canal is surrounded by internal and external sphincter, ischioanal and ischiorectal fossa and it extends from anal verge to levetor ani muscle. The inferior extension of circular smooth muscles of rectum forms internal anal sphincter. The external anal sphincter is formed by striated muscle which is contiguous with levator ani muscle. There is intersphincteric space between internal and external anal sphincter. The anatomical anal canal extends from the perineal skin to the lineadentate. Surgically, the anal canal extends from the perineal skin to the anorectal ring. ${ }^{1}$ This is the circular upper border of the puborectal muscle which is digitally palpable upon rectal examination. The anorectal ring lies approximately $1-1,5 \mathrm{~cm}$ above the lineadentata. The total length of the surgical anal canal is about 4-5 cm. Graphical representation has been depicted in figure 1.

The anal sphincter is comprised of three layers:

- Internal sphincter: continuance of the circular smooth muscle of the rectum, involuntary and contracted during rest, relaxes at defecation.

- Intersphincteric space.

- External sphincter: voluntary striated muscle, divided in three layers that function as one unit. These three layers are continuous cranially with the puborectal muscle and levator ani

\section{Simple contrast fistulography}

Fistulography is a traditional radiological technique used to define the anatomy of fistulas, yet it is an unreliable technique and is difficult to interpret. In fistulography, the external opening is catheterized with a fine cannula, and a water-soluble contrast agent is injected to define the fistulous tract. Few cases of contrast fistulography has been depicted in figure 2.

It has two major drawbacks:

- Difficult to assess secondary extensions secondary to lack of proper filling with contrast material

- Inability to visualize the anal sphincters and to determine their relationship to the fistula

\section{Ultrasound}

All patients were examined with single highly experienced radiologist in left lateral decubitus position and for females additional examination performed in the dorsal lithotomy 
position with an endovaginal ultrasound exam. The patients were examined through transperineal approach to ascertain the presence of perianal abscess or to evaluate the extra sphincteric course of perianal fistula. In cases with perianal abscess we assessed the size, the volume, the site and extensions of abscess around and within the anal canal wall. Also, we assessed the presence of an inter sphincteric plane or submucosal localized collections. ${ }^{2}$

In cases with perianal fistula we assessed the course, the thickness, the length, the side branches, the degree of surrounding soft tissue changes and eventually the site of crossing the external anal sphincter. In all ultrasound approaches images were acquired in axial and sagittal planes. Final Images were analyzed to assess the whole course of perianal fistula from the position of external opening to the site of internal opening. In figure 3 endoanal ultrasound showing postero-lateral wall trans-sphincteric fistula has been depicted. In cases with no internal opening observed injection of dilute hydrogen peroxide into the track was tried. Patientswere followed up with MR imaging.

40 cases detected with perianal fistulas were evaluated for the whole path of the fistula tract either extra sphincteric or sphincteric course by combining transperineal and Transrectalultrasound. Perianal fistula could be classified according to the etiology, sphincteric course, extrasphincteric course, internal opening varieties, external opening varieties and the expected post-operative risks.

\section{Magnetic resonance imaging}

As multiple medical and surgical treatment options exist, imaging plays a critical role in accurately characterizing perianal fistulas to individualize management strategy. Differences in the classification scheme have been shown to have an impact on prediction of prognosis. Imaging options include fistulography, computed tomography (CT), anal endosonography, and MRI.

MRI classification of perianal fistulae has been significantly associated with clinical outcome, with MRI grades differing significantly between satisfactory and unsatisfactory outcomes. MRI evaluation of perianal fistula has also revealed additional diagnostic information in the preoperative setting, especially for complicated disease.

MRI evaluation and classification of perianal fistulae can be standardized with a high degree of diagnostic accuracy therefore reducing interobserver variability.

MRI imaging of perianal fistulae relies on the inherent high soft tissue contrast resolution and multiplanar display of anatomy by this modality, MRI is especially useful in patients with fistula associated with Crohn's disease and those with recurrent fistulae, as these entities are associated with branching fistulous tracts. T2W images (TSE and fat suppressed) provide good contrast between the hyperintense fluid in the tract and hypointese fibrous wall of fistulae, while providing good delineation of the layers of the anal sphincters. In my experience, axial T2W fat-suppressed images were the most useful for locating the fistulous tract. The exact location of primary tract is most easily visualized on axial images. The present of disruption of the external anal sphincter differentiates a transphincteric fistula from intersphincteric one. The internal opening is best seen in this plane. Gadolinium - enhanced T1W images are useful to differentiate a fluid filled tract from area of inflammation.

The tract wall enhancewhere as central portion is hypointense. Abscesses are also very well depicted on post-gadolinium images. To summarize, evaluation of an enhanced T1W image and fat suppressed T2W images provides most of the details necessary for accurate evaluation of perianal fistulae. The anal canal represents the terminal end of gastrointestinal tract which measures about 2.5 to $5 \mathrm{~cm}$ in length. The detailed anatomy of anal canal is basis for evaluating diseases in perianal region. The function of anal canal is to maintain fecal continence. The internal and external sphincter complexes functions in co-ordinated manner to maintain fecal continence. The internal anal sphincter is involuntary while external anal sphincter is voluntary in action. ${ }^{3}$ The internal anal sphincter is continuation of rectal smooth muscle while external sphincter is connected withlevator ani and puborectalis muscle space between these two sphincters provides route to spread of disease.Violation of the levator plate permits access between the superficial ischoioanal fossa and pelvic pararectal space. ${ }^{4}$

The anal sphincter complex consists of two cylindrical layers separated by a fat-containing intersphincteric space. The understanding of fine perianal anatomical detail is crucial to provide an excellent road map prior to surgery. The anal sphincter complex with soft tissue density with hypointense imaging is seen on MRIs as two concentric rings separated by a sheet of fat appearing hyperintense. To locate the internal opening, we adopt the anatomical surgical description of anal canal, according to "anal clock" (figure-5).

Anal sphincter complex is seen as two concentric rings. MR imaging: inner (+) internal sphincter and outer external sphincter (white dovetailarrows) appear relatively hypointense on all T2-weighted images without fat suppression (AC). Levator ani muscle (B, thick arrows) forms superior boundary of fat-containing ischioanal fossa $\left({ }^{*}\right)$ on either side of anal canal. CT (D-F) of inner internal sphincter (+), outer external sphincter (white dovetail arrows), levator ani muscle (E, thick arrows), and fat-containing ischioanal fossa ( $\left(^{*}\right)$.

The major advantage of MR imaging is its capacity to demonstrate extensions associated with a primary tract. Morphologically, MRI's ability to accurately demonstrate the fistula's extensions may provide an excellent road map prior to surgery.

\section{Classification of perianal fistula}

According to the etiology: Perianal fistula can be classified into cryptoglandular, recurrent cryptoglandular and non cryptoglandular. ${ }^{7}$ Cryptoglandular perianal fistula (342 cases-76\%) can be established by their internal openings situated at level of dentate line or ending within the inter sphincteric plane at level of dentate line.Cases of recurrent cryptoglandular perianal fistula (90 cases-20\%) showed past history of operatve management to previously diagnosed cryptoglandularfistula.Cases with non cryptoglandular perianal fistulas (18 cases-4\%) detected with their internal openings above or below the levels of dentate line and mainly caused by Crohn's disease, tuberculosis, previous anorectal surgery, hiradenitisSuppurativa, Trauma and foreign bodies. $^{8}$ 
According to the sphincteric course: The following types of perianal fistula observed

Submucosal fistulas (9 cases-2\%): were caused by cryptoglandular.Infection or Chron's disease and were depicted in either upper or lower anal canal above or below the level of dentate line where the mucosal ligament present.

Inter sphincteric perianal fistula (Figure 6): May extend downward (type A), upward (type B), upward and suprasphincteric (type C) or circumferential (Horse-shoe fistula or type $\mathrm{D}$ ). In type $\mathrm{A}$ the fistula tract extended in a downward direction to reach the perianal skin, in type B it extended in an upward direction with blind termination or with a second internal opening in the anorectal wall, Type C the supra sphincteric type of perianal fistula in which the track ascend in inter sphincteric plane to above puborectalis level and descend in between the puborectus and levator ani muscles to ischio anal fossa and perianal skin, In type D it extended in the circumferential direction to form a horseshoe fistula with its internal opening at the mid line posteriorly. Posterior inter sphincteric horse-shoe fistula can extend upward to high anal canal level without external opening, but can't extend downward due to the presence of anococcygeal ligament, the horse-shoe fistula tract also can extend to the ischioanal fossa and perianal skin. ${ }^{10}$

Transsphincteric perianal fistula (315 cases-70\%): Can be subclassified into 2 types according to the site where they cross the sphincters. Type A at or above the puborectalis muscle (36 cases- $8 \%$ ) or type B below the puborectalis level (279 cases-62\%). In type A all perianal sphincters including the puborectalis muscle are in danger. In type B the puborectalis muscle and the deep external sphincter are spared. Each of them may show abscess formation at the ischioanal fossa. ${ }^{11}$

Extrasphincteric fistula ( 9 cases-2\%): The fistula track pass outside the sphincters to terminate at anorectal wall, it can be caused by Crohn's disease, recurrent cryptoglandular fistula, TB, pelvirectal abscess and diverticulitis. It could be subclassified into: Type A with blind termination, type B with internal opening in rectal wall, type $\mathrm{C}$ with abscess near rectal wall, type $\mathrm{D}$ with abscess at ischioanal fossa.

Superficial or subcutaneous fistula (9 cases-2\%): Pass from skin to beneath the sphincters edges and the internal opening lies below the dentate line and were seen with cases of previous anorectal surgery or hiradenitis Suppurativa. ${ }^{12}$

\section{According to the varieties of internal opening}

The presence of internal opening: It correctly identified the site of internal opening in $88 \%$ of cases and among the remaining 12\%, 23 cases(5\%) showed no internal opening could be identified at surgery. Internal opening could be present or absent either due to blockage or destruction of internal opening or could be seen as tiny holes.

Level of internal opening: Below the dentate line (11 cases-2.5\%), above the dentate line (45 cases-10\%) or at the dentate line (371 cases- 82.5\%). Fistulas with their internal openings below the dentate line were seen due to hiradenitisSuppurativa, Crohn's disease T.B. Fistulas with their internal openings at the dentate line were cryptoglandular in origin, Fistulas open above the dentate line may be due to Crohn's disease, TB or postoperative recurrence. $^{13}$

Number of internal openings: More than one internal opening could be seen (4cases-1\%) in recurrent cryptoglandular fistulas and in Crohn's disease.

According to the varieties of external opening: External opening could be single or multiple. Multiple external openings could be seen with branching fistula or with multiple fistulas. External opening could be situated very close to the anal verge and suggest intersphincteric fistula or could be away from the anal verge and suggest transsphincteric or extrasphincteric fistula. It could be absent in many cases and suggest sinus tract or fistula in progress $(9 \text { cases- } 2 \%)^{14}$

According to the extra sphincteric course: It Could be kinked, curved, obstructed, branching (18 cases-4\%), nonbranching, and may show abscess formation (9 cases-2\%).

The extra sphincteric course of fistula tract may be abnormally long to communicate with pilonidal sinus tract ( 2 cases), to reach the root of scrotal sac and in one case seen extended to the inner aspect of the thigh.

\section{According to the postoperative risks}

Risky for postoperative recurrence: - (89 cases-20\%)

The followings are at increased risks for recurrence.

- An abnormal extra sphincteric course, like multiple branches, abnormal kinks and blocking of tracks.

- Tracks with no internal opening.

- Certain types of fistulas as horse-shoe fistula, high transsphincteric, extra sphincteric and supra sphincteric fistulas.

Some general conditions as diabetes or Crohn'sdisease $\mathrm{e}^{7-10}$.

Risky for postoperative incontinence:- (84 cases-18.5\%)

The followings are at increased risks:

- Patients > 45 years old due to abnormal sphincteric weakness caused by either increased thickness of internal sphincter or decreased thickness of the external sphincter

- Females are more susceptible to incontinence more than males due to a substantial reduction in squeeze pressure and smaller external sphincter mass.

- Females during the childbearing period due to occult sphincteric injuries and pudendal neuropathy occurring during delivery.

- Certain types of fistulas as high transsphincteric, horseshoe, extra sphincteric and supra sphincteric fistulas due to unavoidable operative danger to the anal sphincters and puborectalis muscle.

Preoperative base line incontinence

Certain etiological factors as Crohn's disease and TB. ${ }^{10-14}$

\section{CONCLUSION}

Radiograph and computed tomography do not provide much details and can be consider as obsolete investigation. The endo-anal ultrasound approach was entirely helpful for evaluating the sphincteric course of fistula, but gives 
no mind about the extrasphincteric course which made magnetic resonance imaging more acceptable technique for evaluating perianal fistula since it presents an estimate about the extrasphincteric course. MR imaging provides precise location of the fistulous track, and its relationship to pelvic floor and the sphincter complex and helps in the identification of secondary tracks and abscesses. Providing good delineation of the layer of anal sphincters.In our experience axialT2 $\mathrm{W}$ fat - suppressed images were the most useful for locating fistulous tracts.Gadolinium enhanced T1W images are useful to differentiate a fluid filled tract from an area of inflammation.

\section{REFERENCES}

1. Bhaya AK, Kumar N. MRI with MR fistulogram for perianal fistula: A successful combination. Clin Gastrointest Magnetom 2007;1:56-9.

2. Halligan Steve, Jaap Stoker. Imaging of fistula in ano. Radiology 2006;239:18-33.

3. Beets-Tan RG, Beets GL, van der Hoop AG, Kessels AG, Vliegen RF, Baeten CG, et al. Pre operative MR imaging of anal fistulas: Does it really help the surgeon. Radiology 2001;218:75-84

4. Barker PG, Lunniss PJ, Armstrong P, Reznek RH, Cottam K, Phillips RK. Magnetic resonance imaging of fistula-in-ano: Technique, interpretation and accuracy. ClinRadiol 1994;49:713.

5. Spencer JA, Chapple K, Wilson D, Ward J, Windsor AC, Ambrose NS. Outcome after surgery for perianal fistula: Predictive value of MR imaging. AJR Am J Roentgenol 1998;171:403-6.

6. Stoker J, Rociu E, Zwamborn AW, Schouten WR, Lameris JS. Endoluminal MR imaging of the rectum and anus: Technique, application and pitfalls. Radiographics 1999;19:383-98.

7. Halligan S, Bartram CI. MR imaging of fistula in ano: Are endorectal coils the gold standard? AJR Am J Roentgenol 1998;171:407-12.

8. Park AG, Gordon PH, Hardcastle JD. A classification of fistula-in-ano. Br J Surg 1976;63:1-12.

9. Morris J, Spencer JA, Ambrose NS. MR imaging classification of perianal fistulas and its implications for patient management. Radographics 2000;20:623-35.

10. Lunnis PJ, Armstrong P, Barker PG, Reznek RH, Philips RK. MR imaging of the anal fistulae. Lancet 1992;340:394-6.

11. Beckingham H, Spencer JA, Ward J, Dyke GW, Adams C, Ambrose NS. Prospective evaluation of dynamic contrast enhanced MRI in evaluation of fistula-in-ano. BulSurg 196;83:1396-8.

12. Lilus HG. Fistua-in-ano, an investigation of human foetal anal ducts and intramuscular glands and a clinical study of 150 patients. ActaChirScandSuppl 1968;383:7-88.

13. Maier AG, Funovics MA, Kreuzer SH, Herbst F, Wunderlich M, Teleky BK, et al. Evaluation of perianal sepsis: Comparison of anal sonography and MRI. J Magn Reson Imaging 2001;14: 254-60.

14. Spencer JA, Ward J, Beckingham IJ, Adams C, Ambrose NS. Dynamic contrast enhanced MR imaging of perianal fistulas. AJR Am J Roentgenol 1996;167:735-41.

\section{Source of Support: Nil; Conflict of Interest: None}

Submitted: 23-07-2019; Accepted: 10-08-2019; Published online: 04-09-2019 